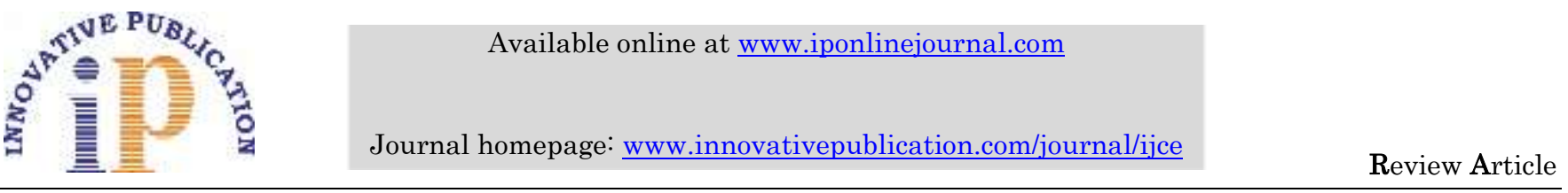

\title{
Oral health considerations in geriatric patients -A review
}

\section{Sheetal Ghivari}

Reader, Dept. of Conservative and Endodontics, Maratha Mandal Dental College, Belgaum, Karnataka, India

\begin{abstract}
Oral health is closely related to general health which needs special attention in elderly patients. In order to provide health care to elderly patients understanding their mental, physical and psychosocial needs is very important. In due course of old age many physiologic changes takes place in many organs of the body including teeth. Considering the oral changes the treatment strategies for elderly patients need to be modified. A thorough counseling is required to bring the change in their thought process that tooth loss is part of ageing process.
\end{abstract}

Keywords: Elderly, Oral health, Tooth loss.

\section{Introduction}

Gerodontology deals with dental problems especially for older patients. In twentieth century longevity of human race has increased throughout world. According to survey average life expectancy at birth increased from 69.8 years for male 72.3 years for female by 2021-2025.

As a result of increased life expectancy, retirement age in many private and government increased up to 70 years. As younger generations in India is facing unemployment problem the retirement age has not been increased in many states. According government of India's survey the people above 60 years and above age are considered elderly and are eligible for various government policies.

This demographic shift in Indian population has led to multitude of health implications. Due to increased life expectancy the community has to bear greater burden of diseases and disability. Among many diseases and disabilities the elderly people undergo dental diseases occupy important place. Due to physiologic ageing the manual dexterity will get affected along with poor muscular tonicity which leads to poor oral hygiene maintenance ultimately causing gingival and periodontal problems, increasing the number of oral pathogenic microorganisms. Future dentists will have to focus on treating elderly patients by making necessary changes and advancements in their clinical practice. ${ }^{3}$

\section{Common dental problems of elderly}

Most commonly identified dental diseases in elderly patients is senile caries, attrition, periodontal diseases, eudentulism, poor quality of alveolar ridges, ill fitting dentures, mucosal lesions, dry mouth and cancers. All these diseases are sequelae of poor oral hygiene maintenance and consumption of diet rich in cariogenic potential as well as lack of awareness regarding preventive aspects and practicing the habits of tobacco and beetle nut chewing. Systemic health problems which are associated coexisting polypharmacy has certain side effects such as xerostomia.oral cavity of elderly is predisposed to many oral diseases due to lack of antimicrobial and immunological function of saliva. The prevailing dental problems in elderly has its deleterious effects on general well being, esthetics, speech and masticati. ${ }^{4}$

The complexity of the treatment increases with increasing age as elderly patients suffer from dementia, psychosis, neurosis, parkinson's disease and many musculoskeletal disorders

Hence ability to seek treatment. Successful management of elderly patient depends upon proper psychological management and the time that the dentists allocate. $^{5}$

Another point worth emphasizing is lack of knowledge in psychological management of elderly people which requires special training. Many people hesitate to work in this field as it requires a lot empathy and interest of wellbeing of elderly patients.

\section{The elderly segment of population}

Elderly patients aged 65-74 are new elderly

$75-84$ years are mid old

85 years and above are oldest old. ${ }^{6}$

\section{Oral health status in elderly}

Nutrition in old age

Adequate nutrition is required for well being of elderly patients, as basic metabolic rate decreases caloric requirement also drops down. Due to ill-fitting dentures and mobile teeth result in reduced interest or ability to eat. Around $8000 \mathrm{kj}$ calorie requirement in 80 years old with $0.97 \mathrm{grm} / \mathrm{kg}$ body weight of protein along with calcium, iron, ascorbic acid and potassium are required. ${ }^{7}$

*Corresponding Author: Sheetal Ghivari, Dept. of Conservative and Endodontics, Maratha Mandal Dental College, Belgaum, Karnataka, India

Email: kubasadsheetal@yahoo.in

http://doi.org/10.18231/j.ijce.2019.019 


\section{Changes in salivary glands and secretions}

Diminished function of salivary gland seen most commonly after ageing due to decreased acinar content. The main oral health problems of old age that dryness of mouth and root caries (senile caries) are caused by reduced salivary flow. ${ }^{8}$

\section{Age changes in oral mucous membranes}

Oral mucous membrane has a protective function both epithelium and connective tissues synthesize several substances such as keratin and laminin which are critical for maintenance of mucosal surfaces. As a sign of ageing the mucosa turns to thin, smooth and acquires satin like edematous appearance with loss of surface stipplings.The tongue appears to be bald with loss of filiform papillae and tendency to development of sublingual varices and candidial infections.

Additionally due to use of various prosthesis which considerably alters the mucosal integrity if not maintained properly.

\section{Age changes in teeth}

After eruption of tooth undergoes gradual change over years this is referred as age changes. Enamel lacks the physiological turnover once it is damaged but dentin and cementum continuously get deposited. The enamel of elderly people becomes less permeable and more brittle due to ion-exchange mechanisms.

Wear and attrition are the macroscopic changes which affect the tooth form. Due to loss of perikymata and imbrication lines, the enamel surface appears flat with less detail than in newly erupted teeth. Changes in surface structures cause altered light reflection in older individuals which affects the observed color. Both in quantity (thickness) and quality of dentin, pigmentation of anatomical defects, corrosion products and also inadequate oral hygiene are the factors which affects variation in tooth shades. ${ }^{8}$

Dental pulp of elderly people contain fewer cells and more fibers, reducing the total volume of the pulp. Degeneration of both myelinated and unmyelinated nerve fibres shows less reparative ability as compared to young pulp tissue. Pulp calcifications are also found to increase in frequency, number and size with age.

\section{Ageing and periodontal disease}

Periodontal disease in elderly is due to chronic periodontitis in adulthood, severity of which enhanced by length of time the periodontal tissues have been exposed to the dentogingival bacterial plaque and is considered to reflect the individual's cumulative oral history. Changes in dietary habits, variation in salivary gland secretions may affect the growth of microorganisms. ${ }^{10}$

\section{Prosthetic consideration in geriatric dentistry}

Prosthethetic treatment should be postponed in elderly people suffering from chronic debilitating diseases until general health is restored. Adaptability to prosthetic treatment in elderly people is slow due to degeneration of Functional elements in central nervous system. The outcome of prosthetic treatment is complicated by mental disorders. Due to progressive atrophy of buccal and labial musculature chewing efficiency is not improved. Rampant caries and reduced retention of dentures is a consequence of xerostomia which occurs in medically compromised patients. ${ }^{8}$

\section{Preventive services for elderly}

Many elderly are not exposed to preventive practices as they hold the opinion that tooth loss is a physiologic process of ageing and seek dental treatment only in emergency.

For socioeconomically disadvantaged elderly people, professional dental health services were very low. Preferred method of brushing for most elderly sulcular brushing with soft brush. Patients with gingival recession should use extra soft brush with light pressure and modification of brushing technique. Plaque retention in elderly is exacerbated by presence of restorations and partial dentures. Elderly patients face problems with mechanical removal of plaque because of reduced manual dexterity and impaired vision. Elderly with poor manual dexterity may benefit from the use of traditional mechanical toothbrushes, rotary electric toothbrushes, or manual brushes that have been adapted or customized for each person. ${ }^{11}$

Mouth rinses may contain chlorhexidine, sodium benzoate, sanguinaria, a fluoride, or other remineralizing agents, which can prevent oral disease and should be recommended to the elderly when appropriate.Chlorhexidine has many benefits it is effective against variety of bacteria, it reduces mucositis and candidiasis in immune suppressed patients. Reminerlizing rinses are helpful to an elderly patient who develops repeated root caries. ${ }^{12}$

\section{Denture care}

Edentulous patients should be educated about denture hygiene to prevent and soft tissue protection. The tissues can be prevented from harm by avoiding constant wearing of dentures. Removal of dentures while retiring to bed at night and proper insertion and removal technique need to be demonstrated. Cleaning and massaging the tissues atleast once a day enhances the blood circulation. Immersion of dentures in cleansers followed by brushing and cleaning should be instructed. ${ }^{5}$

\section{Counseling and education}

Elderly patients need to be educated and motivated about oral hygiene care. Simple but effective model for providing oral care at home should be taught to patient or else to a care taker. Tell -show -do is the most effective method to train elderly patients which includes explain the procedure, demonstrate the procedure finally elderly can do or practice that technique. ${ }^{5}$

The dentist should try to dispel the misconception of elderly, who think that tooth loss is inevitable consequence 
of aging. Oral health education and screening programs should be organized regularly so that early preventive and therapeutic measures can be taken to prevent the progression of oral diseases in elderly patients.

\section{Conclusion}

Major drawback in providing oral health care to elderly patient is lack of awareness of preserving natural dentition. With changing attitude more emphasis should be placed on keeping the teeth of elderly in healthy state so that they can have pretty smile.

\section{Source of Funding}

None.

\section{Conflict of Interest}

None.

\section{References}

1. Registrar General, India, Report and tables on Age, Series-I, India, C-14, C-14 SC and C14 ST, Vol. I. Census of India 2001: New Delhi; 2004.

2. Registrar General, India, Population Projections for India and States 2002-2006: Report of the Technical Group on PopulationProjection Constituted by the National Commission on Population: New Delhi; 2006.

3. Talwar M, Chawla HS. Geriatric dentistry: Is rethinking still required to begin undergraduate education? Indian J Dent Res 2008;19(2):175-77.
4. Matthiessen PC. Demography-impact of an expanding elderly population. In: Pedersen PH, Loe H, editors. Textbook of geriatric dentistry. 2nd ed. Copenhagen: Munksgaard; 1996;505-27.

5. Razak PA, Jose Richard KM, Thankachan RP, Abdul Hafiz K A , Nanda Kuma K, Sameer K M et al. Geriatric Oral Health: A Review Article. J Inter Oral Heal 2014;6(6):110-6.

6. National Programme for the Health Care of the Elderly (NPHCE). Directorate General of Health Services Ministry of Health \& Family Welfare Government of India.

7. 7.Soini H, Routasalo P, Lauri S, Ainamo A. Oral and nutritional status in frail elderly. Spec Care Dent 2003;23:20915.

8. 8.Vissink A, Spijkervet FK, Amerongen VA. Aging and saliva: A review of the literature. Spec Care Dent 1996;16(3):95-103.

9. 9.Papas AS, Niessen LC, Chauncey HH. Geriatric Dentistry Aging and Oral Health. St. Louis: Mosby Yearbook; 1991.

10. World Health Organization. WHO Oral Health Country/Area Profile. Available from: http://www. whocollab.od.mah.se/index.html. [Last accessed on 2014 Oct 03].

11. Schou L, Wright K. Danish public awareness of general health and international perspectives. Tandlaegebladet 982;86(15):511-6

12. Persson RE, Truelove EL, LeResche L, Robinovitch MR. Therapeutic effects of daily or weekly chlorhexidine rinsing on oral health of a geriatric population. Oral Surg Oral Med Oral Pathol 1991;72(2):184-91.

How to cite this article: Ghivari S. Oral health considerations in geriatric patients -A review. Indian $J$ Conserv Endod 2019;4(3):83-5. 